Palavras chave: Ergonomia Saúde Silvicultura

Histórico: Recebido |4/I2/20 I I Aceito $21 / 10 / 2013$

Keywords: Ergonomics Health Slviculture

Correspondência: eslopes@pq.cnpq.br
Felipe Martins de Oliveira', Eduardo da Silva Lopes², Carla Krulikowski Rodrigues²

\section{AVALIAÇÃO DA CARGA DE TRABALHO FíSICO E BIOMECÂNICA DE TRABALHADORES NA ROÇADA MANUAL E SEMIMECANIZADA}

RESUMO: Nesta pesquisa, objetivou-se realizar uma avaliação da carga de trabalho físico e biomecânica de trabalhadores nas atividades de roçada manual e semimecanizada para a implantação de plantios florestais. A carga de trabalho físico foi avaliada por meio do levantamento da frequência cardíaca dos trabalhadores nas diversas fases do ciclo de trabalho, com uso de um monitor Polar Oy. A avaliação biomecânica foi realizada por meio de filmagens dos trabalhadores no trabalho, sendo definidas as posturas típicas e os dados analisados no programa 3DSSPP (Programa de Predição de Postura e de Força Estática), desenvolvido pela Universidade de Michigan, EUA. Os resultados mostraram que, a atividade de roçada manual requereu maior esforço físico, com carga cardiovascular acima do limite máximo recomendado de $40 \%$, sendo a atividade classificada como pesada. Nesse método de trabalho, várias articulações dos trabalhadores apresentaram riscos de lesões, sendo o quadril a articulação mais comprometida, com percentual de capazes variando entre 88 e $92 \%$.

\section{EVALUATION OF THE PHYSICAL WORK LOAD AND BIOMECHANICAL OF WORKERS AT MANUAL AND SEMI-MECHANIZED MOWING}

ABSTRACT: The objective of this research was to evaluate of the physical work load and biomechanical of workers at manual and semi-mechanized mowing activities for the planting the forests. The physical work load was evaluated in a survery of the workers cardiac frequency in different stages of the work using a Polar Oy monitor. To the biomechanic evaluation the workers were filmed in the execution of the work, defined the typical postures adopted and the date analyzed in program 3DSSPP (3D Static Strength Prediction Program) developed for University of Michigan, USA. The results showed that the activity of manual mowing required greater physical effort, with cardiac frequency above the maximum limit recommended of $40 \%$ and the activity was classified as heavy. In this method of working the various joints of the workers showed injurie risks. The hip joint articulation was the most affected, with capable percentage varying between 88 and $92 \%$. 


\section{INTRODUÇÃO}

O setor florestal brasileiro apresenta uma grande importância para a sociedade em termos econômicos, sociais e ambientais. Atualmente, a área plantada com florestas é de aproximadamente 6,7 milhões de hectares, sendo $76,6 \%$ com plantios de Eucalyptus e $23,4 \%$ de Pinus, devendo ressaltar que as perspectivas apontam que até 2020, a área plantada deverá atingir a 10 milhões de hectares (ASSOSIAÇÃO BRASILEIRA DE PRODUTORES DE FLORESTAS PLANTADAS - ABRAF, 20I3).

Ao contrário da área de colheita de madeira que possui máquinas e equipamentos de alta tecnologia e produtividade, as atividades de implantação florestal, especialmente a roçada é realizada por meio de métodos manuais ou semimecanizados e envolvendo um grande contingente de mão de obra, além de afetar a qualidade e produtividade do trabalho.

Além disso, diversos autores relatam que nas atividades de implantação florestal, os trabalhadores podem exercer atividades de elevado esforço físico, adotar posturas potencialmente lesivas e manusear cargas com peso acima dos limites toleráveis (FIEDLER et al., 2007; SILVA et al., 2007; TOUPIN et al., 2007). Tais situações, segundo lida (2005), poderão comprometer a produtividade, causar o desconforto, aumentar os riscos de acidentes e danos à saúde dos trabalhadores.

Nesse contexto, surge a análise ergonômica do trabalho, que busca melhor entender como o trabalho está sendo realizado sob o ponto de vista do esforço físico e das posturas adotadas, subsidiando a tomada de decisão para a melhoria das condições de conforto, segurança e saúde dos trabalhadores.

A carga de trabalho físico (CTF) expressa a quantidade de carga máxima que um indivíduo pode suportar em determinadas condições de trabalho, por meio de aferição de índices fisiológicos, determinandose a duração e as pausas de trabalho. É útil ainda em estudos comparativos, visando a obter os esforços físicos nos diferentes métodos ou sistemas de trabalho, podendo ser obtida por meio do dispêndio energético ou de índices fisiológicos como a frequência cardíaca (COUTO, 1995; GRANDJEAN, 1982). A medição da frequência cardíaca tem sido utilizada por vários autores para estimar a carga de trabalho física dos trabalhadores em diferentes tipos de trabalho (KIRK; SULLMAN, 200I; SULLMAN; BYERS, 2000; TOUPIN et al., 2007).

Outro aspecto a ser considerado é a ocorrência de lombalgias nos trabalhadores que atuam na área florestal, sendo normalmente frequentes e causadas pela adoção de posturas inadequadas durante o levantamento e a movimentação de cargas em atividades realizadas de forma contínua (FIEDLER et al., 2007; LOPES; OLIVEIRA, 20II).

Fiedler e Venturoli (2002) dizem, ainda, que na área florestal comumente ocorrem fadigas por sobrecarga física, com os trabalhadores adotando posturas inadequadas e gerando dores no sistema musculoesquelético, tendo como conseqüência, a redução do ritmo de trabalho e raciocínio, que pode levar aos erros, e até mesmo, ao seu afastamento por doenças ocupacionais. Já, Silva et al. (2007) afirmam que, quando o trabalhador adota uma postura forçada por períodos prolongados, existe o risco eminente de sobrecarga mecânica, acarretando quadros álgicos e desequilíbrios de força.

Diante disso, objetivou-se, neste trabalho, realizar uma avaliação da carga de trabalho físico e biomecânica dos trabalhadores nas atividades de roçada manual e semimecanizada para a implantação de plantios florestais.

\section{MATERIAL E MÉTODOS}

Este estudo foi realizado em uma empresa prestadora de serviços florestais, localizada na região do Norte Pioneiro, Paraná, entre as coordenadas geográficas $24^{\circ} 0$ I' $58^{\prime \prime}$ de latitude sul e $50^{\circ} 27^{\prime} 28^{\prime}$ ' de longitude oeste, altitude média de 776 metros.

O clima predominante da região é, segundo classificação de Köppen, subtropical úmido mesotérmico - Cfa, com temperatura média anual de $21^{\circ} \mathrm{C}$ e precipitação média anual entre 1.200 e $1.400 \mathrm{~mm}$.

Os dados foram coletados nos meses de janeiro e fevereiro de 2010, quando o Índice de Bulbo Úmido e Termômetro de Globo (IBUTG) médio era de $31^{\circ} \mathrm{C}$., em áreas de reforma para a implantação de um plantio do híbrido Eucalyptus grandis $x$ Eucalyptus urophylla, enquanto o relevo foi classificado como suave a ondulado.

Foi estudada a atividade de roçada pré-plantio em uma área com cobertura de vegetação herbáceoarbustiva composta pelas seguintes espécies: Matayba elaeagnoides (miguel-pintado), Allophylus edulis (vacum), Zanthoxylum rhoifolium (mamica-de-cadela), Acacia bonariensis (nhapindá) e Jacaranda micrantha (carobinha).

Foram estudados os métodos de roçada manual e semimecanizada (Figura I). No manual, foi utilizada uma foice com cabo de madeira de I,I5 metros de comprimento e massa total de $1,5 \mathrm{~kg}$, enquanto no semimecanizado uma roçadora da marca Stihl, modelo FS 220, com massa de 7,7 kg e comprimento de I,85 m, sem o conjunto de corte, motor de I,7 kW de potência e equipada com lâmina de três pontas de $300 \mathrm{~mm}$. 

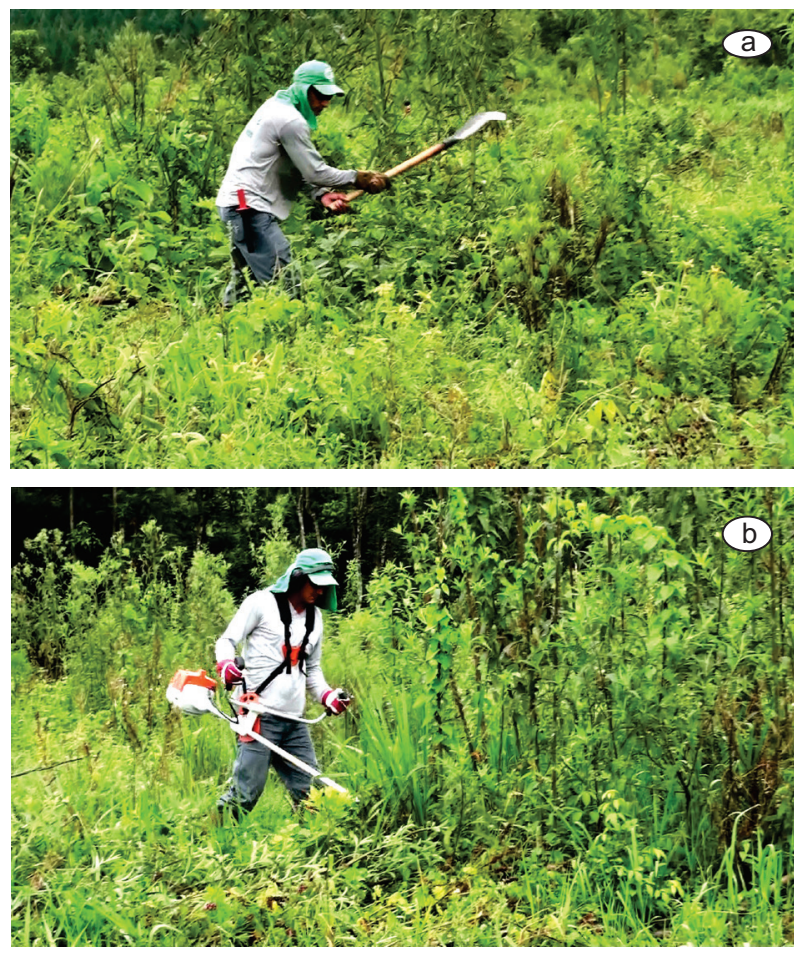

FIGURA I Roçada manual (a) e semimecanizada (b) avaliada no estudo.

FIGURE I Manual (a) and semi-mechanized (b) mowing evaluated in the study.

A atividade de roçada manual foi dividida nas fases: roçada propriamente dita, manutenção da ferramenta, deslocamento e pausas, enquanto a roçada semimecanizada dividida em: roçada propriamente dita, manutenção do equipamento, deslocamento, abastecimento e pausas.

O estudo foi realizado com uma amostra de seis trabalhadores treinados e com experiência em ambos os métodos de trabalho, executando o trabalho dentro de um ritmo normal e com produtividade semelhante, possibilitando, assim, a comparação dos métodos de trabalho (Tabela I).

TABELA I Características dos trabalhadores participantes da pesquisa.

TABLE I Characteristics of workers participating in the research.

\begin{tabular}{lcc}
\hline \multirow{2}{*}{ Característica } & \multicolumn{2}{c}{ Roçada } \\
\cline { 2 - 3 } & $32 \pm 6$ & $32 \pm 6$ \\
\hline Idade (anos) & $73,1 \pm 7,9$ & $73,1 \pm 7,9$ \\
Peso (kgf) & $1,68 \pm 0,05$ & $1,68 \pm 0,05$ \\
Estatura (m) & $0,30 \pm 0,07$ & $0,29 \pm 0,06$ \\
$\begin{array}{l}\text { Produtividade média } \\
\text { efetiva }\left(\text { ha } \cdot \mathrm{h}^{-1}\right)\end{array}$ & \\
\hline
\end{tabular}

Todos os trabalhadores receberam esclarecimentos sobre os objetivos e a metodologia da pesquisa, por meio da leitura e assinatura do Termo de Consentimento Livre e Esclarecido (TCLE), atendendo à Resolução n 196/96 da Comissão Nacional de Ética em Pesquisa (CONEP) do Ministério da Saúde.

Para a avaliação da carga de trabalho físico, foi realizado, incialmente, um estudo piloto buscando definir o número mínimo de repetições dentro de cada método de trabalho, utilizando a equação I, proposta por Conaw (1977), sendo $\mathrm{n}=$ número mínimo de ciclos operacionais necessários; $\mathrm{t}=$ valor de $\mathrm{t}$, para o nível de probabilidade desejado e (n-I) graus de liberdade; $C V=$ coeficiente de variação, em porcentagem; e $\mathrm{E}=$ erro admissível, em \%.

$$
n \geqslant \frac{t^{2}+C V^{2}}{E^{2}}
$$

Como os dados de frequência cardíaca dos trabalhadores foram obtidos em intervalos de 5 segundos, obteve-se um total de 2.502 e 2.445 amostras por trabalhador nos métodos manual e semimecanizado, respectivamente, perfazendo, assim, um total de 12.510 e 12.225 amostras de frequência cardíaca, atendendo ao mínimo necessário.

A área experimental totalizou 7,56 hectares, sendo a mesma subdividida em dois blocos de 3,78 hectares com características semelhantes para a execução de cada método de trabalho. Posteriormente, cada bloco foi subdividido em seis parcelas de 0,63 hectares, onde cada trabalhador executou um método de trabalho. A subdivisão da área experimental serviu para orientar os trabalhadores na realização das atividades e facilitar a coleta dos dados a campo.

A avaliação da carga de trabalho físico foi realizada por meio da determinação da carga cardiovascular. Para tal, foi obtida a frequência cardíaca média dos trabalhadores na execução das diversas fases do trabalho (FCT) e a frequência cardíaca média em repouso (FCR), obtida antes do início do trabalho, sendo ambas, as médias aritméticas dos batimentos cardíacos coletados durante o período amostrado.

Foi utilizado um monitor de frequência cardíaca da marca Polar e modelo RS800CX, que foi instalado nos trabalhadores no início do trabalho e retirado ao final, sendo os dados de frequência cardíaca captados em intervalos de cinco segundos. Ao final, os dados foram transferidos e analisados em software específico.

A carga cardiovascular (CCV) foi obtida por meio da equação 2 (APUD, 1989), sendo $\mathrm{CCV}=$ carga cardiovascular (\%); FCT = frequência cardíaca medida durante $\circ$ trabalho; $F C R=$ frequência cardíaca de 
repouso medida no início da jornada de trabalho; e FCM = frequência cardíaca máxima (220 - idade).

$$
\mathrm{CCV}=\frac{\mathrm{FCT}-\mathrm{FCR}}{\mathrm{FCM}-\mathrm{FCR}} \cdot 100
$$

Quando a carga cardiovascular ultrapassa o limite de $40 \%$, trata-se de uma situação em que o trabalhador está com sobrecarga física, sendo necessário calcular a frequência cardíaca limite e o tempo de repouso (pausas), por meio da equação 3 , sendo $\mathrm{Tr}=$ tempo de repouso (min); FCL: frequência cardíaca limite; e $\mathrm{Ht}=$ tempo de trabalho ( $\mathrm{min}$ ).

$$
\operatorname{Tr}=\frac{\mathrm{Ht} \cdot(\mathrm{FCT}-\mathrm{FCL})}{\mathrm{FCT}-\mathrm{FCR}}
$$

Paralelamente, foi realizado um estudo de tempos e movimentos empregando-se $\bigcirc$ método de cronometragem de tempos contínuos. Foram determinados os tempos consumidos nas diferentes fases da roçada em ambos os métodos de trabalho, sendo, em seguida, relacionados com as frequências cardíacas dos trabalhadores. Foi utilizado um cronômetro centesimal e formulários de campo específicos desenvolvidos para essa finalidade.

Ao final do estudo, foi determinada a carga de trabalho físico exigida em cada fase da atividade, a duração das atividades e fases do trabalho, as pausas necessárias e classificado o trabalho, conforme metodologia proposta por Apud (1989).

Para a avaliação biomecânica, inicialmente foram realizadas filmagens dos trabalhadores em diversos ângulos na execução do trabalho. Após o processamento das imagens, verificou-se que os trabalhadores da roçada manual adotavam quatro posturas típicas, enquanto da roçada semimecanizada adotavam três, correspondendo a 95 e $97 \%$ do tempo total de permanência na fase de roçada propriamente dita, respectivamente.

As imagens das posturas típicas foram então "congeladas" e inseridas no software para a realização da modelagem 3D e avaliação biomecânica por meio da análise das forças aplicadas nas diversas articulações do corpo (pulsos, cotovelos, ombros, tronco, quadril, joelhos e tornozelos) e no disco L5-SI (vértebras Lombar 5 e Sacral I) da coluna vertebral. Foi utilizado o software 3DSSPP (3D Static Strength Prediction Program - Programa de Predição de Postura e de Força Estática 3D), versão 6.0.5, desenvolvido pela Universidade de Michigan, Estados Unidos.

A análise do software forneceu a carga limite recomendada, que corresponde ao peso que pelo menos $99 \%$ dos homens consegue manusear. A carga limite recomendada induz a uma força de compressão de $3.426,3 \mathrm{~N}$ sobre o disco L5-SI da coluna vertebral que pode ser tolerada pela maioria dos trabalhadores em boas condições de saúde.

\section{RESULTADOS E DISCUSSÃO}

\section{Avaliação da carga de trabalho físico}

$\mathrm{Na}$ Tabela 2, é apresentada a carga de trabalho físico dos trabalhadores por meio da frequência cardíaca. De modo geral, a atividade de roçada manual requereu

TABELA 2 Carga de trabalho físico exigida na roçada manual e semimecanizada.

\begin{tabular}{|c|c|c|c|c|c|c|c|c|}
\hline Atividade & Fase do ciclo & $\begin{array}{c}\mathrm{FCR} \\
(\mathrm{bpm})\end{array}$ & $\begin{array}{l}\text { FCT } \\
(\mathrm{bpm})\end{array}$ & $\begin{array}{l}\text { FCM } \\
(\mathrm{bpm})\end{array}$ & $\begin{array}{l}\mathrm{CCV} \\
(\%)\end{array}$ & $\begin{array}{c}\mathrm{FCL} \\
(\mathrm{bpm})\end{array}$ & $\begin{array}{c}\text { TR } \\
(\min )\end{array}$ & $\begin{array}{l}\text { Classificação } \\
\text { do trabalho }\end{array}$ \\
\hline \multirow{5}{*}{$\begin{array}{l}\text { Roçada } \\
\text { Manual }\end{array}$} & Roçada propriamente dita & \multirow{4}{*}{73} & 133 & \multirow{4}{*}{188} & 52 & \multirow{4}{*}{119} & 112 & $\mathrm{P}$ \\
\hline & Manutenção & & 116 & & 37 & & - & MP \\
\hline & Deslocamento & & III & & 34 & & - & MP \\
\hline & Pausas & & 94 & & 19 & & - & $\mathrm{L}$ \\
\hline & Média / Total & & 126 & 188 & 35 & 119 & 112 & $\mathrm{P}$ \\
\hline \multirow{6}{*}{$\begin{array}{l}\text { Roçada } \\
\text { Semimecanizada }\end{array}$} & Roçada propriamente dita & \multirow{5}{*}{73} & 123 & & 44 & \multirow{5}{*}{119} & 38,4 & MP \\
\hline & Abastecimento & & 106 & & 29 & & - & MP \\
\hline & Deslocamento & & 106 & 188 & 29 & & - & MP \\
\hline & Manutenção & & 102 & & 24 & & - & MP \\
\hline & Pausas & & 100 & & 26 & & - & L \\
\hline & Média / Total & & 116 & 188 & 30 & 119 & 38,4 & MP \\
\hline
\end{tabular}

TABLE 2 Physical effort required in the manual and semi-mechanized mowing.

FCR: frequência cardíaca média em repouso; FCT: frequência cardíaca média em trabalho; FCM: frequência cardíaca média máxima (220 - idade); CCV: carga cardiovascular; FCL: frequência cardíaca limite; TR: tempo de repouso recomendado em função da jornada de trabalho; L: Trabalho Leve; MP: Trabalho Moderadamente Pesado; e P: Trabalho Pesado. 
maior esforço físico dos trabalhadores, sendo classificada como pesada, enquanto no método semimecanizado, a atividade foi classificada como moderadamente pesada, com cargas cardiovasculares médias abaixo do limite máximo de 40\% (APUD, 1989).

Em relação ao método manual, a fase de maior esforço físico foi a roçada propriamente dita, com FCT média de 133 bpm e CCV média de $52 \%$, valores acima dos limites recomendados, sendo a fase classificada como pesada. Tal resultado mostrou a ocorrência de sobrecarga física nos trabalhadores e necessidade de reorganização ergonômica do trabalho, por meio do estabelecimento de rodízios de funções, melhoria dos métodos e ferramentas de trabalho, etc. Caso tais medidas não possam ser adotadas, recomenda-se a adoção de pausas de recuperação de 14 minutos por hora trabalhada.

É importante ressaltar que, as pausas de recuperação devem ser definidas por meio da organização ergonômica do trabalho, pela necessidade de alternância entre esforço e repouso, pois o organismo humano necessita de período de recuperação de energia para que possa manter a sua capacidade funcional (APUD, 1989). Além disso, Silva et al. (2007) comentam que as pausas são necessárias para evitar a sobrecarga do trabalho, quando se detectam excessos de carga física.

No método semimecanizado, a fase de roçada propriamente dita também exigiu maior esforço físico, com FCT média de 123 bpm e CCV média de $44 \%$, cujos valores estiveram pouco acima dos limites admissíveis. As demais fases foram classificadas de leve a moderadamente pesada e não apresentaram sobrecarga física, contribuindo, ainda, para amenizar o maior esforço físico da fase de roçada propriamente dita.

$\mathrm{Na}$ Figura 2, são mostradas as oscilações de frequência cardíaca de um mesmo trabalhador atuando em ambos os métodos de roçada. Nota-se que houve reduções pontuais momentâneas da frequência cardíaca em intervalos de aproximadamente 30 minutos ao longo do trabalho, ocasionadas pelas pausas pessoais e manutenção da ferramenta. Entretanto, verifica-se que a frequência cardíaca durante todo o período esteve acima do limite recomendado de I 9 bpm, evidenciando o maior esforço físico exigido pelo método de roçada manual comparado ao semimecanizado.

Essas reduções na roçada manual mostram que a diversificação das atividades ao longo da jornada de trabalho contribuiu para a redução da CTF e amenização do esforço físico. Entretanto, nota-se que a frequência cardíaca durante todo o período manteve-se acima do
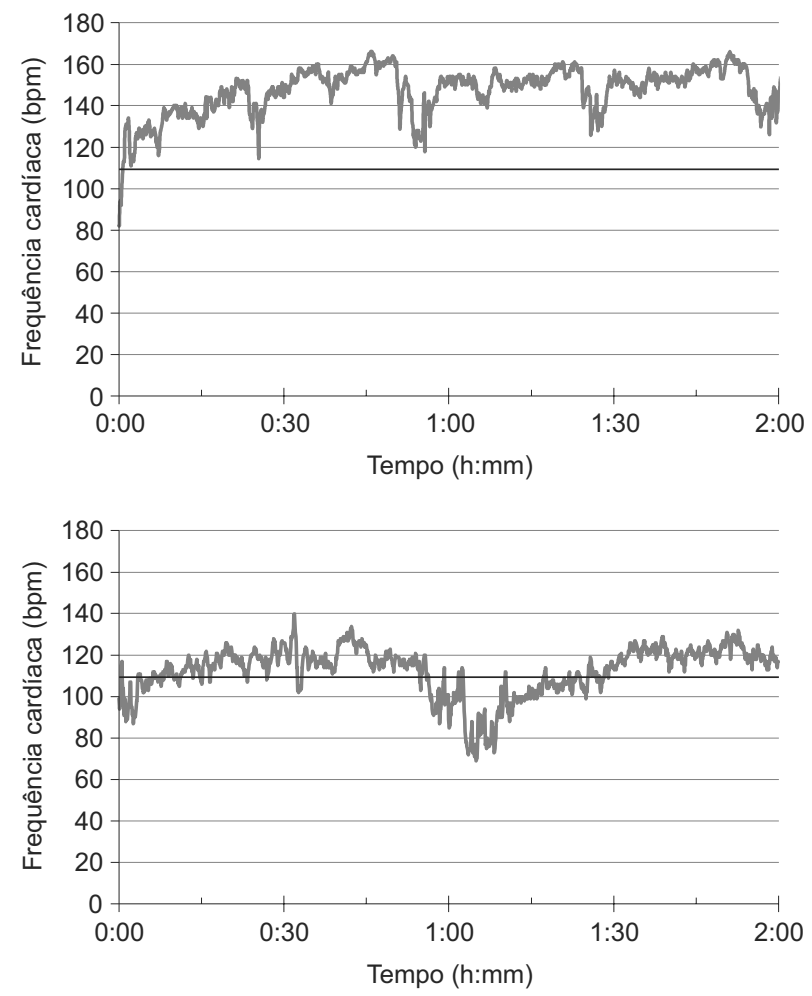

FIGURA 2 Frequência cardíaca observada de um trabalhador nas atividades de roçada manual (a) e semimecanizada (b) em função do tempo.

FIGURE 2 Heart frequency observed of a worker in the activities of manual (a) and semi-mechanized (b) mowing in time function.

limite recomendado de $119 \mathrm{bpm}$, evidenciando o maior esforço físico exigido pelo método de roçada manual comparado ao semimecanizado.

Em relação ao método semimecanizado, houve uma redução nos batimentos cardíacos do trabalhador após uma hora trabalhada, em razão da alternância de fases durante a realização da atividade. É importante ainda ressaltar que, a frequência cardíaca ficou em torno de 110 bpm, evidenciando o menor esforço físico do método.

\section{Avaliação biomecânica}

$\mathrm{Na}$ Tabela 3, são apresentadas as forças de compressão aplicadas no disco L5-SI da coluna vertebral no limite máximo de $3.426,3 \mathrm{~N}$ e o percentual de capazes nas articulações do corpo dos trabalhadores. Todas as posturas típicas foram definidas apenas nas fases de roçada propriamente dita em ambos os métodos, em razão de as mesmas representarem em torno de $80 \%$ do tempo total de trabalho, sendo o tempo restante ocupado pelas demais fases. 
TABELA 3 Força de compressão no disco L5-SI da coluna vertebral no limite máximo de 3.426,3 N e percentual de capazes nas articulações do corpo dos trabalhadores na roçada manual e semimecanizada.

TABLE 3 Compressive strength on the L5-SI column disc in the maximum of 3,426.3 N and percentage of the able joints of workers bod in manual (a) and semi-mechanized (b) mowing.

\begin{tabular}{|c|c|c|c|c|c|c|}
\hline $\begin{array}{l}\text { Métodos de } \\
\text { roçada }\end{array}$ & $\begin{array}{c}\text { Postura típica } \\
\text { na fase de roçada } \\
\text { propriamente dita }\end{array}$ & $\begin{array}{c}\text { Tempo de } \\
\text { permanência na } \\
\text { postura (\%) }\end{array}$ & $\begin{array}{c}\text { Força de } \\
\text { compressão no } \\
\text { disco L5-SI (N) }\end{array}$ & $\begin{array}{c}\text { Risco de } \\
\text { lesão no } \\
\text { disco L5-SI }\end{array}$ & $\begin{array}{c}\text { Articulações } \\
\text { comprometidas }\end{array}$ & $\begin{array}{c}\text { Percentual capazes } \\
\text { com risco de lesão } \\
(\%)\end{array}$ \\
\hline \multirow{4}{*}{$\begin{array}{l}\text { Roçada } \\
\text { manual }\end{array}$} & F1 & 19 & 1.507 & SRL & $\begin{array}{c}\text { Pulsos } \\
\text { Cotovelos } \\
\text { Ombros } \\
\text { Tronco } \\
\text { Quadril } \\
\text { Joelhos } \\
\text { Tornozelos }\end{array}$ & $\begin{array}{c}99 \\
100 \\
99 \\
99 \\
88 \\
98 \\
99\end{array}$ \\
\hline & & 16 & 1.663 & SRL & $\begin{array}{c}\text { Pulsos } \\
\text { Cotovelos } \\
\text { Ombros } \\
\text { Tronco } \\
\text { Quadril } \\
\text { Joelhos } \\
\text { Tornozelos }\end{array}$ & $\begin{array}{c}99 \\
100 \\
99 \\
99 \\
88 \\
97 \\
98\end{array}$ \\
\hline & 3 & 20 & 1.247 & SRL & $\begin{array}{c}\text { Pulsos } \\
\text { Cotovelos } \\
\text { Ombros } \\
\text { Tronco } \\
\text { Quadril } \\
\text { Joelhos } \\
\text { Tornozelos }\end{array}$ & $\begin{array}{c}99 \\
100 \\
99 \\
99 \\
92 \\
96 \\
99\end{array}$ \\
\hline & & 40 & 1.523 & SRL & $\begin{array}{c}\text { Pulsos } \\
\text { Cotovelos } \\
\text { Ombros } \\
\text { Tronco } \\
\text { Quadril } \\
\text { Joelhos } \\
\text { Tornozelos }\end{array}$ & $\begin{array}{c}99 \\
100 \\
99 \\
99 \\
88 \\
97 \\
99\end{array}$ \\
\hline \multirow{3}{*}{$\begin{array}{c}\text { Roçada } \\
\text { Semimecanizada }\end{array}$} & & 23 & 419 & SRL & $\begin{array}{c}\text { Pulsos } \\
\text { Cotovelos } \\
\text { Ombros } \\
\text { Tronco } \\
\text { Quadril } \\
\text { Joelhos } \\
\text { Tornozelos }\end{array}$ & $\begin{array}{c}99 \\
100 \\
99 \\
99 \\
96 \\
96 \\
99 \\
\end{array}$ \\
\hline & -2 & 49 & 363 & SRL & $\begin{array}{c}\text { Pulsos } \\
\text { Cotovelos } \\
\text { Ombros } \\
\text { Tronco } \\
\text { Quadril } \\
\text { Joelhos } \\
\text { Tornozelos }\end{array}$ & $\begin{array}{c}99 \\
100 \\
99 \\
99 \\
96 \\
96 \\
99\end{array}$ \\
\hline & 2 & 25 & 395 & SRL & $\begin{array}{c}\text { Pulsos } \\
\text { Cotovelos } \\
\text { Ombros } \\
\text { Tronco } \\
\text { Quadril } \\
\text { Joelhos } \\
\text { Tornozelos }\end{array}$ & $\begin{array}{c}99 \\
100 \\
100 \\
99 \\
96 \\
96 \\
99\end{array}$ \\
\hline
\end{tabular}

SRL: Sem Risco de Lesão (SRL). 
Como pode ser visto, nenhuma das posturas típicas adotadas pelos trabalhadores impuseram riscos de lesão no disco L5-SI, estando as forças de compressão abaixo do limite máximo de 3.426,3 N. Apesar disso, ficou evidente o maior esforço causado pelo método manual, com força de compressão variando de 1.247 a $1.663 \mathrm{~N}$.

Em relação às articulações, é possível verificar que o quadril foi a articulação mais comprometida em todas as posturas adotadas no método manual, com percentual de capazes de $88 \%$ nas posturas I, 2 e 4 e $92 \%$ na postura 3. Tal ocorrência poderá estar associada à postura mais inclinada, adotada pelos trabalhadores, situação inversa à observada no método semimecanizado. A postura menos inclinada é recomendada no caso de trabalhos em pé, pois, segundo Dul e Weerdmeester (1995), facilita a manutenção da carga próxima ao corpo e oferece menores riscos de lesões.

No método semimecanizado, as únicas articulações comprometidas foram o quadril e os joelhos, que ocorreu em todas as posturas típicas, com percentual de capazes de $96 \%$.

\section{CONCLUSÕES}

O método de roçada manual apresentou maior sobrecarga física nos trabalhadores, sendo a atividade classificada como pesada e havendo a necessidade de melhoria nas condições ergonômicas.

O quadril foi a articulação mais comprometida em ambos os métodos de trabalho, ocasionada pelas posturas inclinadas adotadas pelos trabalhadores, com maior comprometimento na roçada manual.

Apesar dos métodos de trabalho terem ultrapassado o limite máximo recomendado, a roçada semimecanizada ofereceu as melhores condições de trabalho em termos de esforço físico e biomecânicos, comprovando a viabilidade da mecanização das atividades silviculturais.

\section{AGRADECIMENTOS}

Ao $\mathrm{CNPq}$ pelo apoio financeiro, a empresa florestal pelo apoio e cessão da área de estudos e aos trabalhadores participantes.

\section{REFERÊNCIAS}

APUD, E. Guide-lines on ergonomic study in forestry. Genebra: Internacional Labour Office, 1989. 241 p.
ASSOSIAÇÃO BRASILEIRA DE PRODUTORES DE FLORESTAS PLANTADAS. Anuário estatístico da ABRAF 2013: ano base 2012. Brasília, 2013. 148 p.

CONAW, P. L. Estatística. São Paulo: E. Blucher, 1977. 264 p.

COUTO, H. A. Ergonomia aplicada ao trabalho: o manual técnico da máquina humana. Belo Horizonte: Ergo, 1995. v. I, $353 \mathrm{p}$.

DUL, J.; WEERDMEESTER, B. Ergonomia prática. São Paulo: E. Blucher, 1995. 147 p.

FIEDLER, N. C.; FERREIRA, A. H. S.; VENTUROLI, F; MINETTI, L. J. Avaliação da carga de trabalho físico exigida em operações de produção de mudas ornamentais no Distrito Federal: um estudo de caso. Revista Árvore, Viçosa, v. 3I, n. 4, p. 703-708, 2007.

FIEDLER, N. C.; VENTUROLI, F. Avaliação da carga física de trabalho exigida em atividades de fabricação de móveis no Distrito Federal. Cerne, Lavras, v. 8, n. 2, p. I I7-|22, jul./ dez. 2002.

GRANDJEAN, E. Fitting the task to the man: an ergonomic approach. London: Taylor \& Francis, 1982. $379 \mathrm{p}$.

IIDA, I. Ergonomia: projeto e produção. 2. ed. São Paulo: E. Blücher, 2005. 360 p.

KIRK, P. M.; SULLMAN, M. J. Heart rate strain in cable hauler choker setters in New Zealand logging operations. Applied Ergonomics Journal, Guildford, v. 32, n. 4, p. 389-398, 2001

LOPES, E. S.; OLIVEIRA, F. M. Avaliação da carga de trabalho físico e da postura de trabalhadores na produção de mudas florestais. Cerne, Lavras, v. I7, n. 4, p. 573-582, out./dez. 2011 .

SILVA, E. P.; MINETTE, L. J.; SOUZA, A. P. Análise ergonômica do trabalho de coveamento semimecanizado para o plantio de eucalipto. Scientia Forestalis, Piracicaba, n. 76, p. 7783, dez. 2007.

SULLMAN, M.; BYERS, J. An ergonomic assessment of manual planting Pinus radiata seedlings. Journal Forest Engineering, Guildford, v. II, n. I, p. 53-62, 2000.

TOUPIN, D.; LEBEL, L.; DUBEAU, D.; IMBEAU, D.; BOUTHILLIER, L. Measuring the productivity and physical workload of brushcutters within the context of a production-based pay system. Forest Policy and Economics, London, v. 9, p. I046-1055, 2007. 
